\title{
Innovation in ADHD Care Pathway in a DGH Setting
}

\author{
Swasti Shekhar \& Prashant Mani \\ Mid-Yorkshire NHS Trust, Wakefield, UK \\ correspondence to swastishekhar@gmail.com
}

Cite as: Shekhar S \& Mani P. Innovation in ADHD care pathway in a DGH setting. The Physician 6(1):c5 DOI: $10.38192 / 1 \cdot 6 \cdot 1 . c 5$

\section{Background:}

In UK, the prevalence of ADHD in children (age 5 to 16 years) is 3-5\%. Symptoms of ADHD should meet the diagnostic criteria in DSM-5 or ICD-10. Children with ADHD would benefit from improved organisation of care and better integration of child health services, CAMHS and adult mental health services (NICE guidelines 2008). We describe changes made in the ADHD pathway at a DGH hospital to improve the patient journey.

Methods:

1. GP referrals did not contain relevant information, hence referrals from school/SENCO was introduced. To improve the quality of school report and support provided, ADHD study day was organised (attended by $120 \mathrm{SENCO/school} \mathrm{teachers).} \mathrm{2.} \mathrm{ADHD} \mathrm{nurse} \mathrm{specialists} \mathrm{have} \mathrm{been} \mathrm{trained} \mathrm{to} \mathrm{help} \mathrm{with} \mathrm{improved}$ communication and follow up of patients. 3. Qb test (a computerised test to look quantitatively at hyperactivity, impulsivity and inattention) is done to ensure comprehensive assessment. 4. A clinic proforma was developed to ensure compliance with NICE guidelines. 5. A study day was organised for GPs to improve adherence to shared care guidelines. 6. We liaised with Adult ADHD services to improve transition clinics, and CAMHS team to manage co-morbidities.

\section{Results:}

Improved efficiency- Reduction in clinic time needed for a diagnosis (25 new ADHD assessments in 2018 needed 30 clinic appointments, 60 clinic appointments needed in 2015). Reduction in ADHD assessment waiting times to 8 weeks (16-18 weeks in 2015). Patient satisfaction - DNA rates have decreased from $22 \%$ in 2015 to $4-5 \%$ in 2018 . Feedback forms have shown significant improvements. Audit looking at compliance to NICE guidelines (NG87) for assessment, counselling, drug treatment, monitoring and transition has shown an improvement across all parameters. All GP surgeries do prescriptions according to shared care guidelines.

\section{Conclusions:}

The new innovative ADHD care pathway has led to safe, effective, efficient and sustainable delivery of ADHD services. 